\title{
Effects of ethanol on adjunctive drinking and barpressing under various schedules of food reinforcement
}

\section{R. M. GILBERT \\ Addiction Research Foundation, Toronto, Ontario, Canada}

An intraperitoneal dose in the order of $1.0 \mathrm{~g} / \mathrm{kg}$ was required to eliminate the FR 24 performance of rats; whereas doses higher than $1.5 \mathrm{~g} / \mathrm{kg}$ were usually required to eliminate barpressing under FI 20-sec or DRL 12-sec schedules. The dose required to eliminate adjunctive drinking was usually less than that required to eliminate barpressing. Efficient DRL performance was dependent upon drinking in the undrugged state but often occurred without drinking after ethanol administration.

During an unpublished investigation of a possible schedule-dependent tolerance effect of ethanol (of the kind reported by Schuster, Dockens, \& Woods, 1966), rat barpressing on a FR 24 schedule was far more affected by a moderate dose of ethanol than barpressing on a DRL 12-sec schedule, even though the former schedule provided a higher reinforcement rate without the drug. Both schedules engendered adjunctive drinking from a freely available water bottle. The amount of such drinking was also reduced by ethanol, often at a lower dose than that required to change the rate of barpressing. The present study provides additional data on these phenomena, including data from rats that barpressed on a FI $20-\mathrm{sec}$ schedule.

\section{METHOD \\ Subjects}

Eight male hooded rats were used, each aged about 4 months at the beginning of experimentation.

\section{Apparatus}

Two similar experimental chambers were used. One was a standard Grason-Stadler (Gerbrands) two-bar rat chamber, equipped with a $45-\mathrm{mg}$ pellet dispenser and contained in its regular enclosing chest. The left bar was removed and a drinking bottle was mounted inside the chamber with its metal spout near the center of the door. The other chamber was similar in essential features to the Grason-Stadler chamber. It was not enclosed and differed in that, to provide for closed-circuit TV monitoring, the back and the side opposite the drinking bottle sloped outward at $45 \mathrm{deg}$. Both drinking bottles were wired to enable the counting of licks. The bar in each case required a force of approximately $0.20 \mathrm{~N}(21 \mathrm{~g})$ to operate the associated microswitch. Recording and programming were arranged by transistorized modules located in a nearby room and supplemented by counters and cumulative recorders.

\section{Procedure}

Each animal was deprived of food and subsequently maintained at close to $85 \%$ of its original weight by supplementary feeding after experimentation. Daily 1-h sessions were run until rates of barpressing under the respective schedules (see Table 1) were each usually within $\pm 5 \%$ of the previous day's value. Thereafter, each day two $30-\mathrm{min}$ sessions were run, separated by $30 \mathrm{~min}$. Ethanol or saline, if administered, was injected intraperitoneally $15 \mathrm{~min}$ after the end of the first session. No injection was given on Mondays. If performance on a Monday was within a prescribed range (based on previous performance), saline was given on Tuesday. If Tuesday's performance was similarly adequate, a scheduled dose of $10 \%$ ethanol in saline was given on Wednesday. The saline-alone volume was equal to the scheduled ethanol-in-saline volume. Inadequate performance with or without saline led to repetition of the respective condition. If performance was inadequate during the repetition, the next condition (saline or drug) was implemented, but the data were ignored. Occasionally a saline day also followed a Wednesday or Thursday drug day to check for an order effect. Occasionally, too, following adequate performance by DRL animals on a Monday, no saline was administered on Tuesday. Instead, the water bottle was removed for the second session to assess the contribution of adjunctive licking to DRL performance. Initially the ethanol doses were presented in an ascending series $(0.5,0.75,1.0 \ldots 2.5 \mathrm{~g} / \mathrm{kg})$, terminating the series or omitting values as appropriate, both to familiarize the animals with the larger injection volumes (as much as $7.5 \mathrm{ml}$ ) and to assess the useful dose range for each animal. These drug data are not presented. Subsequently each of the doses employed was presented two or more times in an irregular order. Injection volumes greater than $5 \mathrm{ml}$ were administered in two injections, one on each side of the peritoneal area.

\section{RESULTS}

The general effect of ethanol was to reduce the rates of both licking and barpressing. Figure 1 indicates a graded dose-effect relationship for each kind of behavior. The ratios plotted in Fig. 1 take into account both sessions on drug days and both sessions on saline days in the following manner. If the rates during the first and second sessions of a drug day were D1 and D2, and during the first and second sessions of the preceding saline day were $\mathrm{S} 1$ and $\mathrm{S} 2$, respectively, the drug/baseline ratio plotted in Fig. 1 was:

$$
\frac{\mathrm{D} 2 \cdot \mathrm{S} 1}{\mathrm{~S} 2 \cdot \mathrm{D} 1}
$$

This provides a correction (S1/D1) for the simpler D2/S2 drug/baseline comparison. The correction takes into account possible day to day rate changes for causes other than the drug. An indication of the baseline performance of each animal is given in the center three columns of Table 1 .

Considering first the difference between schedules, it is clear that both barpressing and licking were more sensitive to ethanol when the former was maintained by a FR schedule than when it was maintained by either of the other schedules. Barpressing rates were at or close to zero when the $1 \mathrm{~g} / \mathrm{kg}$ dose was administered to the FR animals, whereas at least $1.5 \mathrm{~g} / \mathrm{kg}$ were required to eliminate the barpressing of the other animals. In five of the six FI and DRL animals, a dose of $2.0 \mathrm{~g} / \mathrm{kg}$ did not eliminate barpressing. A few animals pressed at higher 


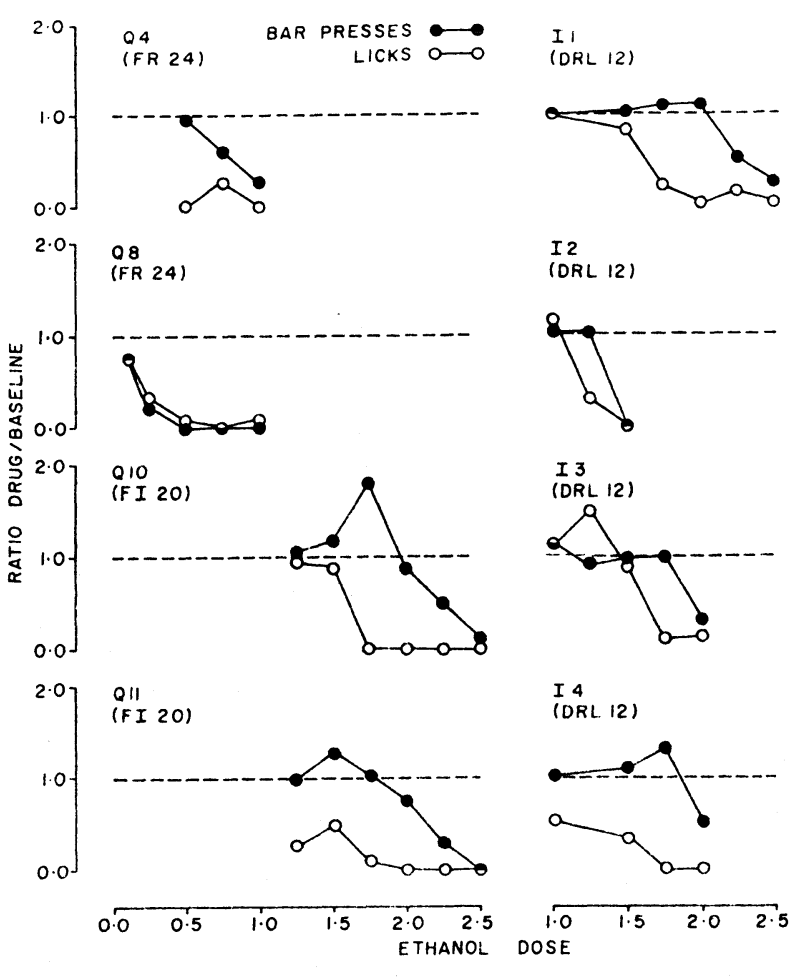

Fig. 1. Drug/baseline ratios for barpress and lick rates for various doses in grams/kilogram of ethanol. The computation of the ratios is described in the text. The dashed horizontal lines indicate ratios of unity. Points lying on or near these lines indicate less drug effect than points lying farther away.

doses even though there was obvious motor impairment. At $2.5 \mathrm{~g} / \mathrm{kg}$ Rat I1 continued to press the bar on occasion while lying prostrate with his snout in the receptacle of the pellet dispenser.

In both FI animals and in two of the DRL animals, a moderate ethanol dose $(1.5-1.75 \mathrm{~g} / \mathrm{kg})$ actually increased rates above baseline values.

One of the FR animals (Q8) was extraordinarily sensitive to ethanol. A dose of $0.25 \mathrm{~g} / \mathrm{kg}$ reliably produced a clear behavioral effect. (This animal was also especially sensitive to sodium pentobarbital, a $5 \mathrm{mg} / \mathrm{kg}$ intraperitoneal dose of which completely suppressed both licking and barpressing. Both drugs could have irritated chronic inflammation of the peritoneal cavity, but none was found on examination and an idiopathic central effect may be presumed.)

With the exception of this rat, Q8, the rate of licking by each animal was more sensitive to ethanol than the rate of barpressing. Figure 1 shows that for each of these animals there was a dose by which licking was substantially affected (i.e., reduced below $50 \%$ of baseline) but by which barpressing was hardly affected (i.e., it was within $15 \%$ of baseline). An alternative formulation of the difference is presented in the two right-hand columns of Table 1 , where it can be seen that the estimated dose required to produce a $50 \%$ decrease in barpressing rate was usually well in excess of the dose required to produce a $50 \%$ decrease in licking rate.

The effect of removing the water bottle on undrugged DRL performance is illustrated in Fig. 2. Comparison of the upper two histograms shows that the effect was to shift the distribution of interresponse times toward shorter values, reducing the proportion and absolute number of reinforced responses. It follows that the licking induced by the DRL schedule contributed to the adequate spacing of barpresses by the rat. The usual effect of the $2.0 \mathrm{~g} / \mathrm{kg}$ ethanol dose was to reduce licking rates to zero or near zero. However, often the overall barpressing rate and the reinforcement rate were hardly affected by the drug, as happened in the session on which the third histogram from the top is based. Thus, it appears that under the influence of the drug, licking was not essential for the spacing of barpresses. Inspection of the actual IRT distribution, however, suggests that the drug had three separate effects. The first was to increase the proportion of very short $(<2 \mathrm{sec})$ IRTs. The second was to reduce the modal IRT. The third was to increase the proportion of long $(>18 \mathrm{sec})$ IRTs. The first and second effects could have been the result of the drug-induced absence of drinking; the third effect could have been the result of the sedative effects of ethanol. In

Table 1

Baseline Rates of Licking, Barpressing, and Reinforcement for Each Rat*

\begin{tabular}{|c|c|c|c|c|c|c|}
\hline \multirow[b]{2}{*}{ Rat } & \multirow[b]{2}{*}{ Schedule } & \multicolumn{3}{|c|}{ Per 30-Min Session } & \multicolumn{2}{|c|}{$\begin{array}{c}\text { Estimated Dose }(\mathrm{g} / \mathrm{kg}) \\
\text { Producing } 50 \% \\
\text { Reduction }\end{array}$} \\
\hline & & Licks & Presses & ments & Licks & Presses \\
\hline Q4 & FR 24 & 663 & 2760 & 115 & $<0.50$ & 0.70 \\
\hline Q8 & FR 24 & 2417 & 1584 & 66 & 0.20 & 0.20 \\
\hline Q10 & FI 20 & 3424 & 522 & 88 & 1.60 & 2.25 \\
\hline Q11 & FI 20 & 2306 & 526 & 87 & 1.25 & 2.10 \\
\hline I1 & DRL 12 & 3630 & 138 & 97 & 1.60 & 2.25 \\
\hline 12 & DRL 12 & 1710 & 141 & 85 & 1.20 & 1.35 \\
\hline 13 & DRL 12 & 1252 & 119 & 97 & 1.60 & 1.90 \\
\hline 14 & DRL 12 & 1547 & 120 & 91 & 1.05 & 2.00 \\
\hline
\end{tabular}

*Shown in each case is the mean rate based on all saline sessions in which adequate performance occurred $(10<N$ $<20$ ). Also shown for each rat are the estimated doses that would have produced a 50\% reduction in licking and barpressing, respectively. 
the session under consideration, these possible effects may have cancelled out each other to provide response and reinforcement rates that were similar to the undrugged state. The bottom histogram in Fig. 2 represents data from an earlier session in which drinking was also (virtually) eliminated by the drug. The three effects observed in the other $2.0 \mathrm{~g} / \mathrm{kg}$ session are also evident in these data, the modal shift being considerably more pronounced. The net result in this session was to produce an increase in barpressing rate and a reduction in reinforcement rate. Here it seems that the increase in response rate consequent upon the elimination of licking was not counteracted by the sedative effect on barpressing.

\section{DISCUSSION}

\section{Schedule-Dependent Effect of Ethanol}

Fixed-ratio performance is sometimes regarded as being relatively insensitive to drug effects. This view is also challenged, recently by Weiss \& Gott (1972), but not to the extent that FR performance is regarded as more sensitive than performance controlled by time-based schedules such as FI and DRL. Hence the unusual nature of the present results. They are not consistent with those of Holloway \& Vardiman (1971), who found similar dose-effect functions for ethanol on FR 20 and DRL 20 performances, both being only moderately depressed at $1.2 \mathrm{~g} / \mathrm{kg}$ and considerably depressed at $1.6 \mathrm{~g} / \mathrm{kg}$. Also, by contrast, Laties $\&$ Weiss (1962) noted that the DRL 20 -sec rate of rats was halved by a $1.0 \mathrm{~g} / \mathrm{kg}$ injection of ethanol, even though the temporal structure of the DRL performance was largely unaffected.

Thus, the FR performance in the present study seems more sensitive to the effects of ethanol, and the DRL performance less sensitive, than might be expected from previous work. There seem to be no comparable reports of the effect of ethanol on FI performance. Comparisons of FI and FR performance when affected by the usually similarly acting drug, sodium pentobarbital, indicate that the FI performance is more sensitive (e.g., Herrnstein \& Morse, 1956).

Two features of the present study might account for the relatively high doses required to affect the DRL and FI performances. One is the high reinforcement rates possible here compared with those of other studies. Reinforcement rate in itself does not determine sensitivity; if it did, the greater sensitivity under the FR schedule in the present study would not have occurred. However, within a given type of schedule it is possible that the drug effect depends upon reinforcement availability.

The second distinctive feature was the availability of a drinking tube. This may have facilitated optimal DRL performance and preserved it during drug action, but it is difficult to see how the drinking could have rendered FI performance less sensitive without having the same effect on FR performance.

One feature that could contribute to increased FR performance sensitivity to a drug, compared with performance under time-based schedules, is the force required to operate the manipulandum. Comparative data are not available. However, it is likely that the much greater work requirement per reinforcement renders FR performance more subject to effort-drug interactions than FI or DRL performances.

\section{Adjunctive Drinking}

In the present study, licking was found to be more sensitive to the effects of ethanol than barpressing. No comparable effect of alcohol has been found before, although two reports (Falk, 1964; Wuttke \& Innis, 1972) have shown that barbiturate administration reduces adjunctive drinking. A FI schedule is

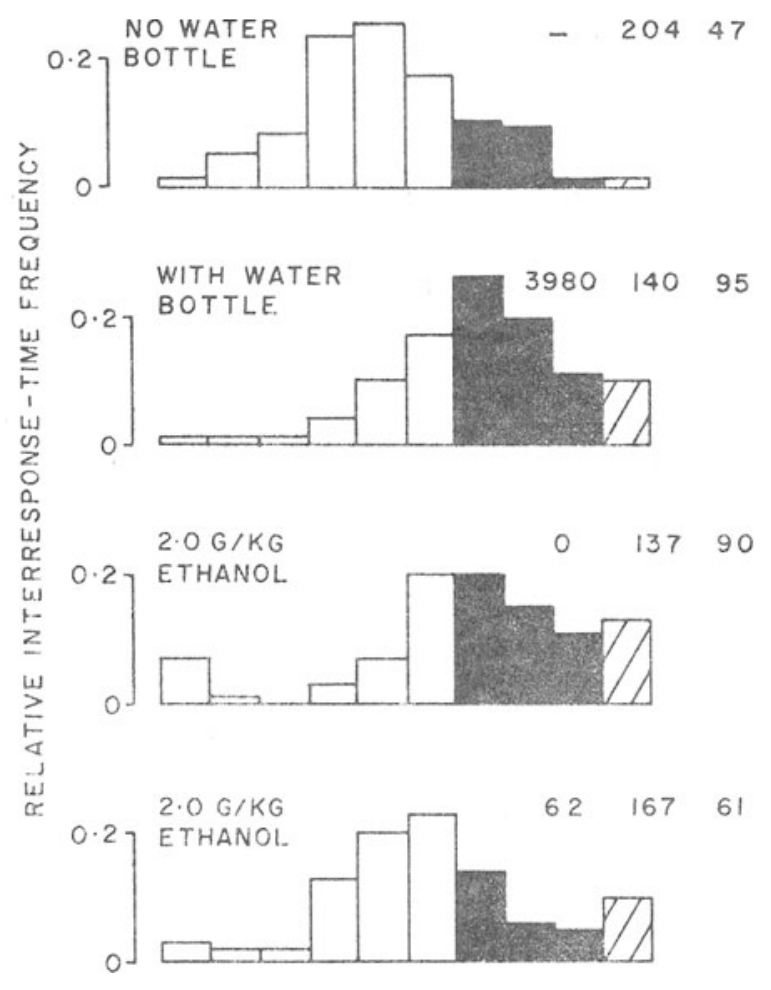

Fig. 2. Relative interresponse-time (IRT) frequencies of barpressing by Rat II during four sessions of the kinds indicated. The open and filled bars represent relative frequencies for 2 -sec bins from 0 to $18 \mathrm{sec}$. Only barpresses represented by filled bars $(12-18 \mathrm{sec})$ and hatched bars $(>18 \mathrm{sec})$ were reinforced. The three numbers at the right of each histogram indicate for that session, from left to right, respectively, the number of licks, the number of barpresses, and the number of reinforcements.

often used with success to induce ethanol drinking (Falk, Samson, \& Winger, 1972), but the session durations are not long enough to allow an effect of the consumed drug.

There are three reasons why ethanol might reduce adjunctive drinking. Two can be ruled out. The first is that ethanol has a direct effect on water consumption. It does of ten have an effect, but this is invariably to increase the amount of water consumed (see review by Wallgren \& Barry, 1970, pp. 534-535). The second reason is that ethanol has an effect on the behavior controlled by the maintaining schedule, an incidental effect of which is to reduce the amount of drinking. For example, if the rate of barpressing were to increase, licking might be excluded. Alternatively, a reduction in barpressing rate might indicate loss of contact with the maintaining contingencies that also reduces the amount of drinking. For most rats in the present study, there was an ethanol-dose at which barpressing rates were little affected, and yet licking was substantially reduced. It follows that this kind of reason does not provide a sufficient account.

The third, and more plausible, reason is that ethanol can reduce the effect of the schedule that gives rise to adjunctive drinking without necessarily affecting the schedule-controlled behavior. Schedule-induced behavior is frequently regarded as a response to a thwarting or frustrating and presumably stressful situation (Falk, 1972). Ethanol has been found to reduce avoidance behavior and also to attenuate the effects of intermittent reinforcement (see review by Wallgren \& Barry, 1970 , pp. 362-363). If adjunctive drinking may be regarded as avoidance behavior, or as behavior controlled by similar features of the environment, its reduction by ethanol would be consistent with these effects. A further indication that adjunctive drinking 
resembles avoidance behavior is provided by Bryant and Falk's unpublished finding, mentioned in Falk (1972), that such drinking is relatively difficult to discourage by punishment contingencies, compared with deprivation-induced drinking. Although a comparative study has yet to be made, it seems to be the case that avoidance behavior is more resistant to punishment than behavior maintained by positive reinforcement.

\section{Dependence of DRL Performance \\ Upon Drinking}

This matter has already been discussed above because frequent reference to the data presented in Fig. 2 was required. It may be worth making the additional comment, however, that the capacity of ethanol to liberate effective DRL performance from dependence upon adjunctive drinking may be added to the list of beneficial effects of ethanol compiled by Wallgren \& Barry (1970, pp. 803-804).

\section{REFERENCES}

Falk, J. L Studies on schedule-induced polydipsia, In M. J. Wayner (Ed.), Thirst: First international symposium on thirst in the regulation of body water. New York: Pergamon Press, 1964. Pp. 95-116.

Falk, J. L. The nature and determinants of adjunctive behaviour.
In R. M. Gilbert and J. D. Keehn (Eds.), Schedule effects: Drugs, drinking, and aggression. Toronto: University of Toronto Press, 1972. Pp. 148-173.

Falk, J. L., Samson, H. H., \& Winger, G. Behavioral maintenance of high concentrations of blood ethanol and physical dependence in the rat. Science, 1972, 177, 811-813.

Herrnstein, R. J., \& Morse, W. H. Selective action of pentobarbital on component behaviors of a reinforcement schedule. Science, 1956, 124, 367-368.

Holloway, F. A., \& Vardiman, D. R. Dose-response effects of ethanol on appetitive behaviors. Psychonomic Science, 1971, 24, 217-220.

Laties, V. G., \& Weiss, B. Effects of alcohol on timing behavior. Journal of Comparative \& Physiological Psychology, 1962, 55, 85-91.

Schuster, C. R., Dockens, W. S., \& Woods, J. H. Behavioral variables affecting the development of amphetamine tolerance. Psychopharmacologia, 1966, 9, 170-182.

Wallgren, H., \& Barry, H., III. Actions of alcohol. Amsterdam: Elsevier, 1970 .

Weiss, B., \& Gott, C. T. A microanalysis of drug effects on fixed-ratio performance in pigeons. Journal of Pharmacology \& Experimental Therapeutics, 1972, 180, 189-202.

Wuttke, W., \& Innis, N. K. Drug effects upon behaviour induced by second order schedules of reinforcement: The relevance of ethological analyses. In R. M. Gilbert and J. D. Keehn (Eds.) Schedule effects: Drugs, drinking, and aggression. Toronto: University of Toronto Press, 1972. Pp. 129-147.

(Received for publication November 29, 1972.) 